\title{
BMJ Open Study protocol for a nationwide questionnaire survey of physical activity among breast cancer survivors in Japan
}

\author{
Yoichi Shimizu, ${ }^{1,2}$ Katsunori Tsuji, ${ }^{1}$ Eisuke Ochi, ${ }^{1,3}$ Hirokazu Arai, ${ }^{4}$ Ryo Okubo, ${ }^{1}$ \\ Aya Kuchiba, ${ }^{5,6}$ Taichi Shimazu (iD , ${ }^{7}$ Naomi Sakurai, ${ }^{8}$ Tomomi Narisawa, ${ }^{1}$ \\ Taro Ueno, ${ }^{9}$ Hiroji Iwata, ${ }^{10}$ Yutaka Matsuoka (1) ${ }^{1,11}$
}

To cite: Shimizu Y, Tsuji K, Ochi E, et al. Study protocol for a nationwide questionnaire survey of physical activity among breast cancer survivors in Japan. BMJ Open 2020;10:e032871. doi:10.1136/ bmjopen-2019-032871

- Prepublication history for this paper is available online. To view these files, please visit the journal online (http://dx.doi org/10.1136/bmjopen-2019032871).

Received 13 July 2019

Revised 15 November 2019 Accepted 16 December 2019

Check for updates

(c) Author(s) (or their employer(s)) 2020. Re-use permitted under CC BY-NC. No commercial re-use. See rights and permissions. Published by BMJ.

For numbered affiliations see end of article.

Correspondence to Professor Yutaka Matsuoka; matsuoka-psy@umin.ac.jp

\section{ABSTRACT}

Introduction A major concern is that few cancer survivors meet the guidelines for recommended levels of physical activity. No studies have investigated physical activity among breast cancer survivors nationwide in Japan. Therefore, the aims of this study are to identify the levels of physical activity among breast cancer survivors, to examine factors-related physical activity among breast cancer survivors and to identify breast cancer survivors' preferences for and interest in exercise programmes in order to inform the future programme development. Methods and analysis We will administer a crosssectional survey using a self-report questionnaire to breast cancer survivors. At each of 50 facilities selected to include a variety of institutional backgrounds according to the population distribution of different regions throughout Japan, we will consecutively distribute the questionnaire to 30 outpatients who have completed initial treatments, except for hormone therapy. The target sample size is 1500 survivors. We will calculate descriptive statistics for each measurement item and perform univariate and multivariate analyses using outcome measures (eg, physical activity and quality of life) related to physical, psychological, social and environmental factors.

Discussion This is the first nationwide survey of physical activity levels among breast cancer survivors in Japan. Identifying the factors associated with physical activity will help us to develop, disseminate and implement programmes that encourage more survivors to adhere to physical activity guidelines.

Ethics and dissemination The protocol was approved by the Institutional Review Board (IRB) of the National Cancer Center on 11 January 2019 (ID: 2018-295). In addition, many of the participating facilities required ethical approval from their local IRBs, while others did not. Accordingly, approval from the local IRBs of individual facilities was obtained when required. The findings will be disseminated through peer-reviewed publications and conference presentations.

\section{INTRODUCTION}

Achieving substantial survivorship is a priority in Japan's cancer countermeasures. Currently, more than 85000 women are newly diagnosed with breast cancer each year $^{1}$, and with the significant improvements
Strengths and limitations of this study

This is the first nationwide survey in Japan on levels of physical activity among breast cancer survivors.

- Because we selected the facilities according to the population distribution of different regions throughout Japan, the results will reflect regional characteristics throughout Japan.

- We chose facilities with a variety of local area population sizes and institutional backgrounds (size, private or public, general or specialised and teaching affiliation); thus, we expect to identify various factors associated with physical activity among breast cancer survivors.

- Physical activity levels may be overestimated because participants who are more interested in physical activity may be more likely to participate in this study.

in early detection and treatment, the 10-year survival rate is about $80 \%$ overall and exceeds $90 \%$ in patients with localised breast cancer. ${ }^{1}$ Among the growing number of survivors with localised breast cancer, improvement of health-related quality of life (HRQOL) after treatment and prevention of recurrence have become a higher priority.

Some healthy behaviours, such as moderate physical activity, a healthy diet and maintenance of a healthy body weight, may lower the risk of recurrence and improve survival. ${ }^{2}$ In particular, a high level of physical activity is related to the extension of healthy lifespan ${ }^{3-9}$ and improvement of HRQOL among breast cancer survivors. ${ }^{3-9}$ Thus, international and Japanese clinical practice guidelines strongly recommend that survivors maintain high levels of physical activity. ${ }^{810}$ Physical activity is defined as any bodily movement produced by skeletal muscles that results in energy expenditure. ${ }^{11}$ American Cancer Society guidelines recommend at least $150 \mathrm{~min}$ per week of moderate-intensity physical activity (eg, moderate walking or light jogging sufficient 
to induce sweating) or at least 75 min per week of vigorous physical activity (eg, jogging or resistance training). ${ }^{10}$ 'The Japan Breast Cancer Society Clinical Practice Guidelines for Breast Cancer' recommend at least $60 \mathrm{~min}$ per week of moderate-to-vigorous intensity physical activity in addition to the physical activity necessary for daily living. ${ }^{8}$ The reason for the apparent disparity between the recommendations of these two national cancer societies is that the method of setting the standard is group based in the Japanese guidelines and individual based in the American Cancer Society guidelines. For the Japanese general population, the Exercise and Physical Activity Reference for Health Promotion published by the Ministry of Health, Labour and Welfare recommends at least $60 \mathrm{~min}$ per week of moderate-to-vigorous physical activity.

However, few breast cancer survivors meet the recommended levels of physical activity. Previous studies in the USA have found that less than half of survivors meet the recommended levels of physical activity in the American Cancer Society guidelines. ${ }^{12}$ In Japan, less than half of gynaecological cancer survivors visiting outpatient departments at four hospitals engage in any physical activities in their leisure time. ${ }^{13}$ Approximately $70 \%$ of the general population also does not regularly exercise. ${ }^{14}$ Exercise is a subset of physical activity that is planned, structured and repetitive and has the improvement or maintenance of physical fitness as a final or intermediate objective. ${ }^{11}$ Thus, we assume that few breast cancer survivors exercise regularly and maintain high levels of physical activity. However, no studies have systematically investigated actual activity levels in breast cancer survivors in Japan.

It is important to identify the actual physical activity of breast cancer survivors throughout Japan, including those residing in urban and rural areas, as well as the factors that can promote or impede such activity, because some previous studies outside Japan have shown that whether breast cancer survivors can maintain and improve exercise habits is affected by cultural, environmental and personal factors. ${ }^{1516}$ In Japan, no studies have identified factors related to the maintenance of exercise habits in breast cancer survivors.

Accordingly, we created a conceptual model (figure 1) based on the PRECEDE-PROCEED model which stands for the Predisposing, Reinforcing and Enabling Constructs in Educational Diagnosis and Evaluation Policy, Regulatory, and Organizational Constructs in Educational and Environmental Development model, a framework used globally in the field of health promotion and public health. ${ }^{17}{ }^{18}$ This study is a preliminary step in the development of an exercise programme designed to

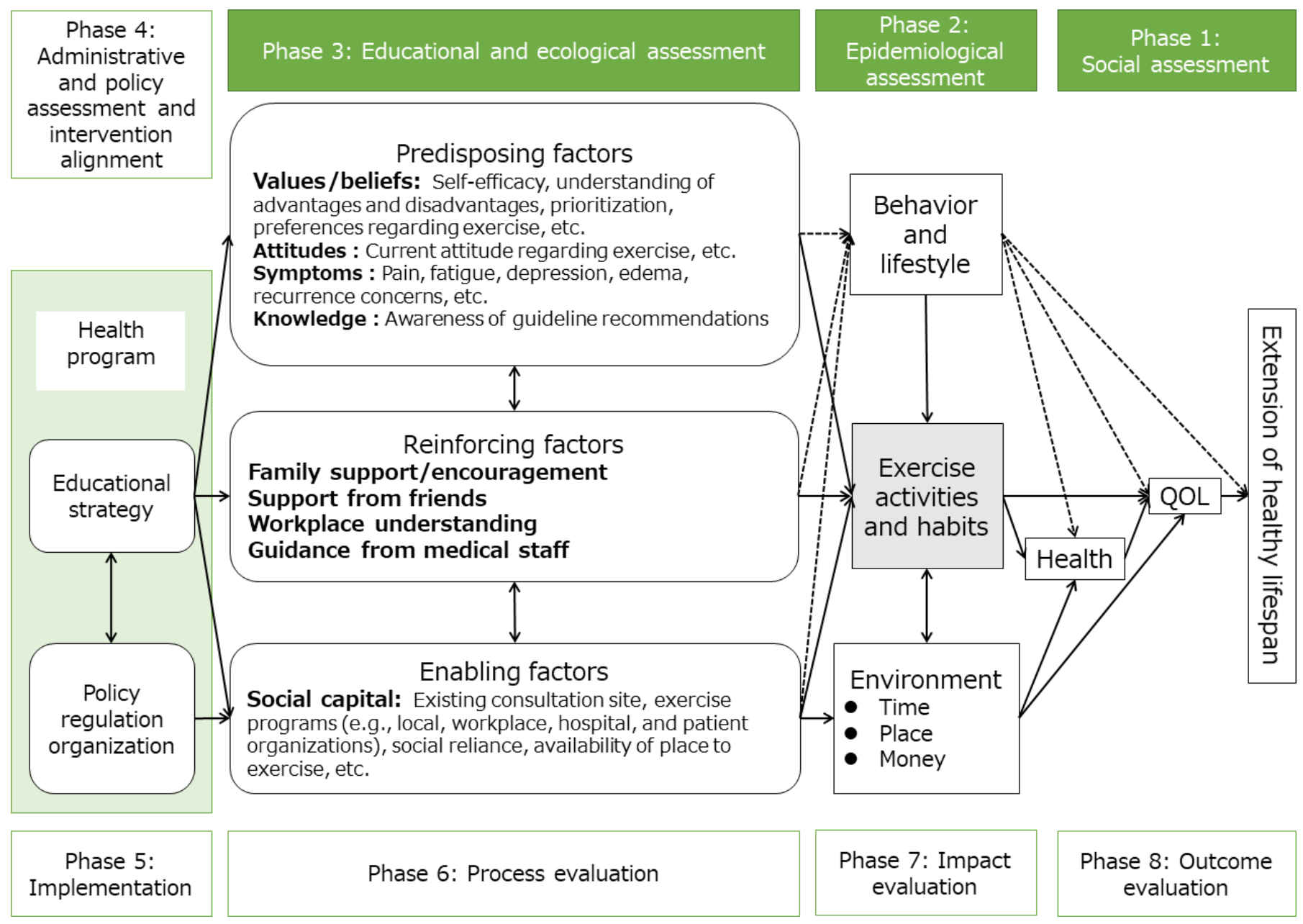

Figure 1 Framework for the study based on the PRECEDE-PROCEED model. Source: Green and Kreuter. ${ }^{18}$ 
empower breast cancer survivors to maintain high levels of physical activity and to determine a pathway for its dissemination and implementation. The study falls under the first to fourth steps of the PRECEDE-PROCEED model. To develop, disseminate and implement-related programmes, it is necessary to identify the factors that impede breast cancer survivors' ability to maintain high levels of physical activity and the factors that make this more likely, in addition to understanding survivors' preferences and interests related to physical activities.

The aims of this study are to identify the levels of physical activity among breast cancer survivors, to examine factors-related physical activity among breast cancer survivors, and to identify breast cancer survivors' preferences for and interest in exercise programmes in order to inform the future programme development.

\section{METHODS AND ANALYSIS}

In this study, we will administer survey questionnaires to breast cancer survivors without recurrence or metastasis within 5 years after treatment for breast cancer. Fifty facilities were selected from among the Japanese Breast Cancer Society accredited facilities ${ }^{19}$ that conduct more than 100 breast cancer surgeries per year. At the outpatient clinic of each facility, we will consecutively deliver the survey forms by hand to 30 breast cancer survivors who are undergoing outpatient care from the survey start date. The target sample size is 1500 survivors. If they complete the forms, they will send them to the research office by mail.

\section{Operational definitions of terminology \\ Cancer survivor}

According to the National Cancer Institute in the USA, a cancer survivor is 'a person who lives after being diagnosed with cancer. Family members, friends and loved ones are also included as 'survivors', as they too are affected'. ${ }^{20}$

The American Cancer Society guidelines offer recommendations regarding physical activity levels divided into three stages: the period during treatment, the period immediately after treatment and the period between the immediate post-treatment period and a stabilised state. ${ }^{21}$ The level of physical activity is expected to differ during each of these stages. Consequently, we focus on the posttreatment period, which is expected to be the main target group for the exercise programme that we intend to develop. Thus, we define a breast cancer survivor as 'a person who has completed treatment for breast cancer, other than hormone therapy among their initial treatments performed for curative purposes, without local progression or recurrence (stage $0-$ IIIa)'.

\section{Maintaining high levels of physical activity}

The Breast Cancer Clinical Practice guideline published by the Japanese Breast Cancer Society recommends that breast cancer survivors maintain high levels of physical activity, which is defined as reporting at least $60 \mathrm{~min}$ of moderate-to-vigorous intensity physical activity a week. ${ }^{8}$

\section{Study sites}

We selected 50 Japanese Breast Cancer Society accredited facilities that conducted more than 100 breast cancer surgeries from April 2016 to March 2017. ${ }^{19}$ Based on the sampling method ${ }^{22}$ for the public opinion survey on cancer countermeasures by the Japan Cabinet Office, we set 22 stratified categories according to the 11 regions (Hokkaido, Tohoku, Kanto, Hokuriku, Tosan, Tokai, Kinki, Chugoku, Shikoku, KitaKyushu and Minami Kyushu) and population sizes (more than 200000 people and fewer than 200000 people) of the cities (and the 23 wards in Tokyo) where facilities are located and decided the number of facilities within each category to match the population distribution. From the list of medical facilities corresponding to each stratified category, facilities were chosen to include a wide variety of institutional backgrounds (size, private or public, general or specialised and teaching affiliation). We will ask each facility to collaborate with this study and to arrange for participants to take the survey.

\section{Participants}

We will consecutively recruit 30 breast cancer survivors who meet the selection criteria from designated dates at the outpatient clinic of each of the 50 facilities. The target sample size is 1500 survivors. Eligibility criteria are as follows: (1) diagnosis of primary breast cancer without distant metastasis, (2) no recurrence, (3) $\geq 20$ years old, (4) completion of initial treatments other than hormonal therapy for curative purposes and (5) the patient has been informed of a diagnosis of breast cancer. Those who cannot respond to the self-reported questionnaire (written in Japanese) are excluded.

\section{Data collection}

In the outpatient clinics of the facilities, attending physicians will hand out a set of materials containing explanatory documents and a survey form to potential participants who meet the selection criteria from the study start dates. They will briefly explain the following: (1) that they should carefully read the letter of intent, (2) that they should not hesitate to contact the research office if they have any questions, (3) that they should tick the agreement column in the questionnaire form if they are able and willing to participate in the survey and (4) that consent is voluntary. If they tick the agreement column, it is considered that appropriate informed consent is obtained. A potential subject will complete questionnaire items independently and send it to the research office. It will take about $40 \mathrm{~min}$ to complete the questionnaire.

\section{Survey items}

In the questionnaire survey, we will measure background information, physical activity levels, factors related to physical activity levels, awareness of the physical activity recommendations in the breast cancer clinical 
practice guidelines, and preferences regarding exercise programmes.

During the preparation of the self-reported questionnaire, we reviewed previous studies and our study group, which consisted of 13 medical personnel and 1 breast cancer survivor, discussed the survey items and our conceptual model (figure 1). We conducted focus group interviews and in-depth personal interviews several times with both medical personnel and breast cancer survivors to explore the associated factors related to breast cancer survivors' physical activity and their preferences for exercise programmes. First, we conducted a focus group interview with medical personnel (two physicians, three nurses and two physical therapists), who had been providing breast cancer survivors with medical care for at least 1 year. Second, we conducted three focus group interviews (two or three breast cancer survivors in each group) and five in-depth semistructured interviews with breast cancer survivors. After recording the interview contents, written transcripts were created. We selected survey items based on the results from qualitative content analysis of these interview data.

After developing the questionnaires, we conducted cognitive checks with five breast cancer survivors to evaluate whether the questions were measuring the construct we intended.

\section{Outcomes}

\section{Physical activity}

The Global Physical Activity Questionnaire (GPAQ) was developed in 2002 as an internationally standardised questionnaire to investigate levels of physical activity and is widely used in policy development by $\mathrm{WHO}^{23-26}$ (table 1). The face validity of the Japanese version of GPAQ has been confirmed. ${ }^{27} 28$ The primary outcome of this study is whether patients' physical activity levels meet the guideline recommendation.

We will also ask about participants' recognition of changes in physical activity levels after diagnosis with a 5-point scale ranging from 'significantly reduced' to 'significantly increased'.

\section{Quality of life}

EuroQol 5 Dimension (EQ-5D) is a comprehensive evaluation scale developed by the EQ group established in 1987 to measure HRQOL. ${ }^{29}{ }^{30}$ We will use the 5-level EQ-5D version, which has improved the instrument's sensitivity and reduced ceiling effects ${ }^{31}$ (table 1). The reliability and validity of the Japanese version have also been confirmed. ${ }^{32}$ The scale consists of five items, and complete health can be converted to 1 and death to 0 as standardised utility values based on the results. ${ }^{32}$

\section{Absenteeism and presenteeism}

We will measure absenteeism and presenteeism with the Japanese equivalent of WHO Health and Work Performance Questionnaire Short Form ${ }^{33-35}$ (table 1). The linguistic face validity of the Japanese version has been confirmed. $^{35}$

\section{Anticipated factors related to physical activity \\ Subject background}

The following attributes of breast cancer survivors will be examined in the questionnaire: age, sex, height, weight, postal code, educational history, employment status, household income, household composition, child care and long-term care situation, free time, treatment history, years since surgery, history of diseases other than breast cancer, use of oral sleeping pills and use of anxiolytics related to relapse concerns. These questions will all be self-reported.

We will ask about participants' past history of exercise at six different ages. Answers range from 'I did not play sports' to 'I exercised 3 or more times a week.' We will also ask about their healthy lifestyle behaviours. For drinking, smoking and eating habits, we created questions based on questions in the lifestyle questionnaire of the National Health and Nutrition Survey. ${ }^{14}$

\section{Symptoms}

\section{Fatigue: Cancer Fatigue Scale}

The Cancer Fatigue Scale (CFS) is a questionnaire designed to measure fatigue in cancer patients, comprising 15 items regarding the patient's sense of fatigue rated on a 5-point scale, from 'not at all' to 'very much.' The scale consists of three subscales: physical malaise, mental fatigue and cognitive fatigue. The reliability and validity of the CFS have been verified ${ }^{36}$ (table 1 ).

\section{Recurrence concerns: Concerns about Recurrence Scale Japanese Version}

Respondents' fear of breast cancer recurrence will be measured by the Concerns about Recurrence Scale (CARS). The CARS consists of 30 items and is used to measure the fear of recurrence in breast cancer patients. ${ }^{37}$ In this study, only four items (overall fear), which are scored on a 6-point scale, will be used as evaluation items. The total score is 4-24 points, and the higher the score, the higher the recurrence fear. The validity of the Japanese version has been confirmed ${ }^{38}$ (table 1$)$.

\section{Insomnia: Japanese version of the Athens Insomnia Scale}

To assess sleep, we will use the Athens Insomnia Scale (AIS), which is an insomnia assessment created by WHO as part of the 'World Sleep and Health Project' that is used globally. ${ }^{39}$ The answers to the eight questions are digitised, with a total score of 24 points, and a score of 6 points or higher is judged to indicate suspicion of insomnia. The validity of the Japanese version (AIS-J) has been confirmed $^{40}$ (table 1).

Other symptoms related to physical activity: US National Cancer Institute Patient-Reported Outcomes version of the Common Terminology Criteria for Adverse Events, Japanese Version

Because outcome assessment by patients themselves has become more important, the Patient-Reported 


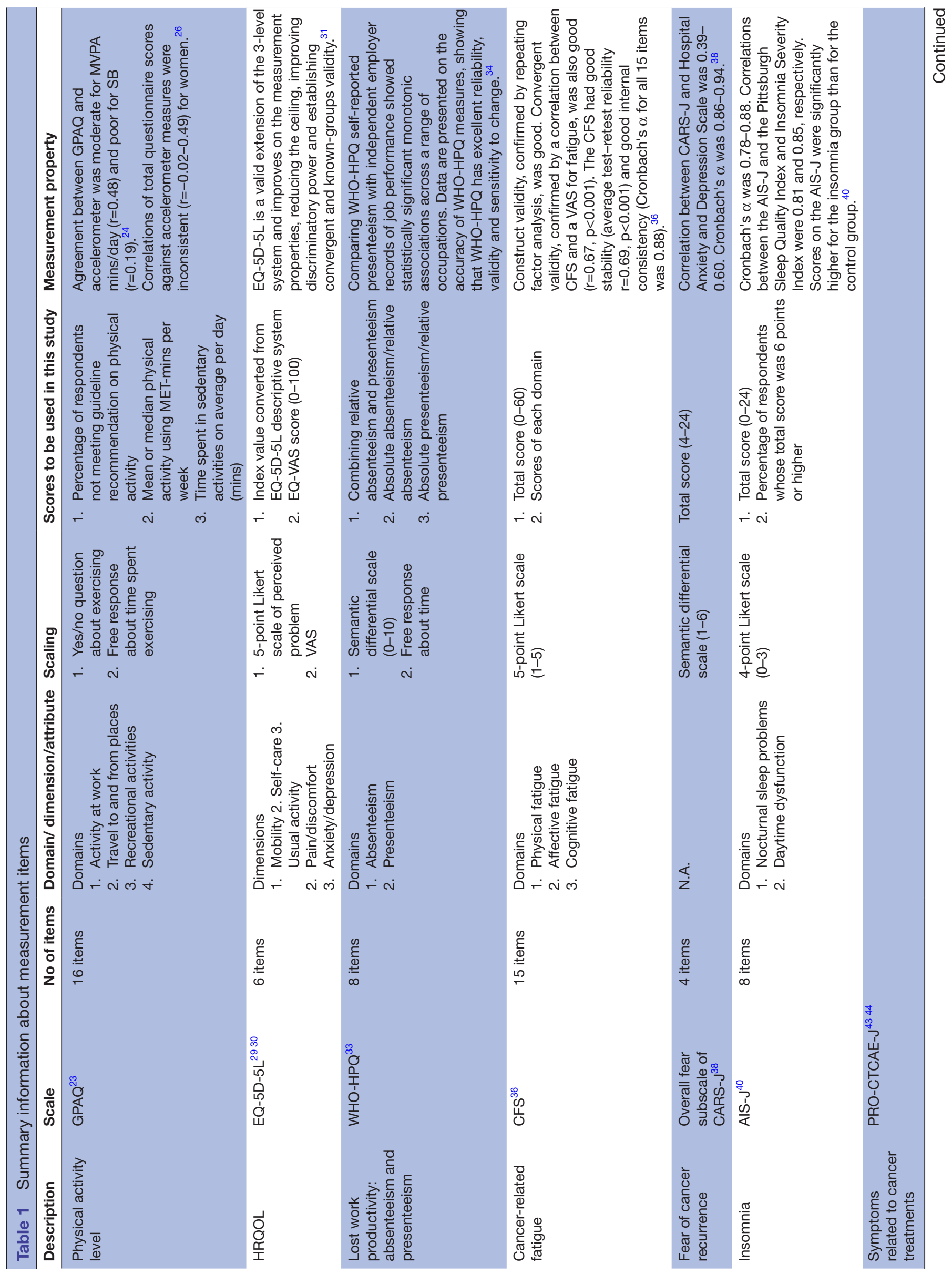




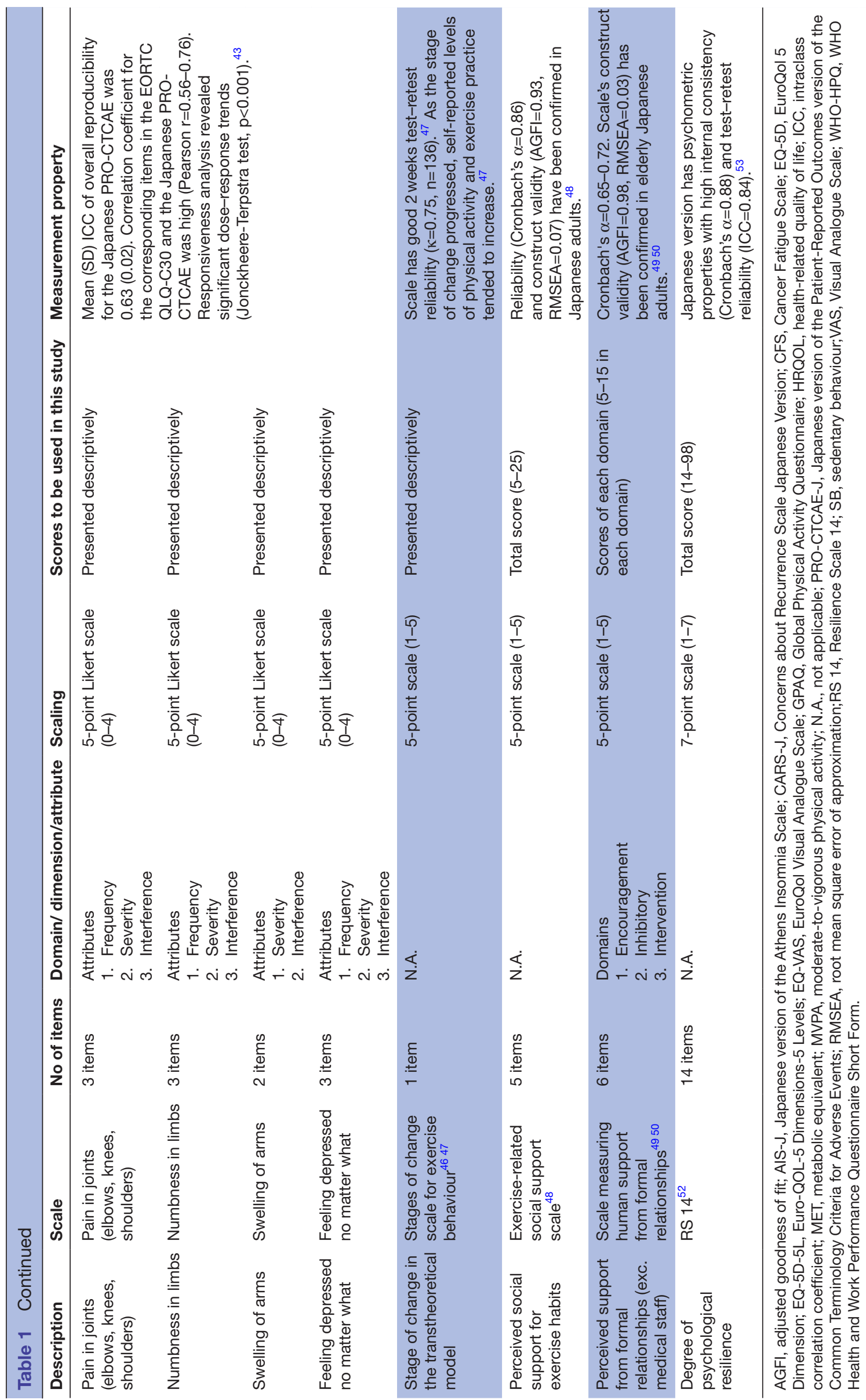


Outcomes version of the Common Terminology Criteria for Adverse Events (PRO-CTCAE) has been developed, and its reliability and validity have been confirmed. ${ }^{41}{ }^{42}$ The validity of the Japanese version of the PRO-CTCAE (PRO-CTCAE-J) has also been confirmed ${ }^{43}{ }^{44}$ (table 1). PRO-CTCAE-J consists of 78 adverse event items, but it is possible to use adverse events partially as needed, and for this study, a relationship has been observed between physical activity in breast cancer survivors and 'pain in joints (elbows, knees, shoulders)', 'numbness in limbs', 'swelling of arms' and 'feeling depressed no matter what'. ${ }^{45}$

\section{Subject characteristics of exercise behaviour}

\section{Stages of change scale for exercise behaviour}

The reliability and validity of the scale developed to measure the stages of change in exercise behaviour based on the transtheoretical model of behavioural change has been confirmed. ${ }^{46} 47$ The patient is instructed to consider personal experiences of and attitudes towards exercise over the past 6 months and choose from a range of options: '1. I currently exercise regularly; I have been doing so for more than 6 months' to ' 5 . I am not exercising, and I will not do so in the future.' Results are classified as a 'period of indifference', 'period of interest', 'preparation period', 'execution period' and 'maintenance period' (table 1).

\section{Exercise-related Social Support Scale}

The Exercise-related Social Support Scale consists of five items (one factor), such as 'family and friends give advice and guidance on how to exercise,' scored on a 5-point Likert scale from 'completely agree (5)' to 'completely disagree (1)' and its reliability and construct validity have been confirmed in Japanese adults ${ }^{48}$ (table 1).

\section{Scale measuring human support from formal relationships}

This is a scale measuring the support received from formal relationships, such as with physicians and nurses, that participants have experienced in the past year. The scale consists of six items scored on a 5-point scale from 'not at all' to 'very much.' The scale's reliability and construct validity has been confirmed in elderly Japanese adults $^{49} 50$ (table 1 ).

\section{Self-efficacy for some exercise programmes}

We will examine the degree of participants' confidence that they can perform possible future exercise programmes. They will choose from '(1) I do not think so' to '(5) I very much think so.'

\section{Preferences for exercise programmes}

Questions about existing programmes and consultation sites

We will ask about questions about participation in existing programmes and consultation sites in free-response form. We will also ask questions using items similar to those in past overseas studies ${ }^{1651}$ about participants' preferences for exercise programmes.
Others

Questions regarding the Japanese Breast Cancer Clinical Practice guidelines

We will ask if they know about the recommendations and the level of evidence for physical activity in Japanese breast cancer clinical practice guidelines.

\section{Resilience Scale 14 Japanese version}

Resilience Scale consists of 14 items that are rated on a 7-point Likert scale to measure degree of psychological resilience. ${ }^{52}$ The Japanese version has psychometric properties of reliability and validity ${ }^{53}$ (table 1 ).

\section{Beliefs about healthy lifestyles}

We will ask about the participants' beliefs about healthy lifestyles and risk of cancer recurrence through four items, which have been prepared with reference to the question items ${ }^{54}$ in the Health Information National Trends Survey (HINTS Survey), regularly conducted by the Division of Cancer Control and Population Sciences of the National Cancer Institute of the USA. These items are reported on a 4-point Likert scale from 'strongly agree' to 'strongly disagree'.

\section{Data analysis}

\section{Statistical analysis}

We will calculate descriptive statistics for the physical activity levels of breast cancer survivors and each measurement item. To identify factors related to meeting the physical activity recommendations, we will perform logistic regression analysis using whether the participants meet recommended physical activity levels as an outcome variable. After performing univariate logistic analysis for each potential-related factor as an explanatory variable, we will perform logistic regression analysis with all related factors as explanatory variables using backward technique by the F-test to identify the significant factors.

Additionally, multiple regression analysis will be also performed with physical activity levels, symptoms and background information as explanatory variables and absenteeism/presenteeism and HRQOL as outcome variables using backward elimination by the F-test to identify significant factors.

A $p<0.05$ for two-sided tests will be considered significant, and we will perform the analysis using SAS V.9.4 (SAS Institute). We will use multiple imputation to handle missing values.

\section{Sample size estimation}

The proportion of people with established exercise habits in the National Health and Nutrition Survey in 2008 was $20 \%-40 \%^{14}$; thus, the percentage of breast cancer survivors who have recommended levels of physical activity is as assumed to be approximately $20 \%$. We calculated the number of samples required to estimate the proportion with margins of error of $\pm 2.5 \%$ ( $\alpha=0.05,984$ people). Therefore, the questionnaire must be distributed to 1500 people if the effective response rate is assumed to be about $70 \%$. Fifty facilities are scheduled to be contacted 
for cooperation and 30 people at each facility will be given the questionnaire.

A sample size of at least $200+(20 \times$ number of explanatory variables) is required for multiple regression analysis. ${ }^{55}$ We will perform logistic regression analysis with about 25 explanatory variables and multiple regression analysis with about 15 explanatory variables. We calculated the required sample size as $200+(20 \times 25)=700$. Thus, the target sample size (1500 people) is estimated to be sufficient for logistic analysis.

\section{Patient and public involvement statement}

We involved a breast cancer survivor in the design of this study protocol as a researcher and coauthor. She discussed issues with other survivors in instances where survivors' preferences and opinions should be considered. In the process of developing the research items, 13 breast cancer survivors informed us of their experiences, priorities and preferences through focus group interviews and in-depth personal interviews. We conducted cognitive checks and a preliminary confirmation of the feasibility, safety and burden of the questionnaires with five breast cancer survivors.

\section{Dissemination}

We will present our findings at medical conferences and submit the results obtained for publication in a peerreviewed international journal.

\section{DISCUSSION}

This study is Japan's first nationwide cross-sectional survey of physical activity in breast cancer survivors. This study is intended to identify the physical activity levels and the factors related to those in Japanese breast cancer survivors. Identifying the associated factors will help us to develop, disseminate and implement programmes to encourage more breast cancer survivors to develop exercise habits and to maintain high levels of physical activity.

It is important to identify the actual physical activity of breast cancer survivors throughout Japan, including those residing in urban and rural areas, as well as the factors that can promote or impede such activity. Factors associated with maintaining and improving exercise habits may vary by culture, environment and survivors' backgrounds. According to a qualitative study by Hefferon et al, age, cancer treatments, existence of comorbidities, fatigue, occupational status, women's traditional roles (household chores, nursing care, etc), poor access to exercise facilities, and seasonal weather changes are factors that could reduce the likelihood of maintaining exercise habits. ${ }^{15}$ In addition, according to a telephone interview survey about barriers to exercise habits carried out by Ottenbacher et al, lack of time, lack of motivation, being discouraged by bad weather, hesitance to exercise alone and feeling as if there is no suitable space to exercise have been highlighted as barriers to exercise. ${ }^{16}$ There may be factors specific to Japan, in particular, walking distances in Japan are generally longer due to the development of public transportation facilities in Japan compared with the largely car-oriented societies seen in Europe and the USA. However, while dense urban populations in Japan use public transportation, there are still many situations where people travel by car, particularly in rural areas.

If this study reveals that breast cancer survivors have low physical activity, it will be necessary to develop, disseminate and implement programmes based on the associated factors, which will be identified in order to encourage more survivors to acquire exercise habits and to maintain high levels of physical activity.

This study has several strengths and limitations.

\section{Strengths}

The first strength is that participants are recruited according to the population distribution of different regions throughout Japan. Therefore, the results will reflect regional characteristics, including cultural, social, ecological and environmental aspects, throughout Japan. The second is that differences can be evaluated based on both institutional backgrounds (size, private or public, general or specialised and teaching affiliation) and the surrounding environment because we selected facilities with various backgrounds from rural and urban areas.

\section{Limitations}

The first limitation is that participants who are interested in physical activity may participate and complete questionnaires more readily than those who are not; thus, the study may overestimate physical activity levels. The second is that use of a self-reported measure of physical activity may overestimate or underestimate participants' physical activity. Previous studies have found that the correlations of total questionnaire scores against accelerometer measurements were inconsistent $(r=-0.02-0.49)$ for women ${ }^{26}$ and that there was moderate agreement between GPAQ and accelerometer measurements for moderate-vigorous physical activity (mins/day) and poor agreement for sedentary behaviour. ${ }^{24}{ }^{26}$ Cleland $e t$ al reported that the higher validity of GPAQ in higherincome countries was likely due to high education levels. ${ }^{24}$

\section{Author affiliations}

${ }^{1}$ Division of Health Care Research, Center for Public Health Sciences, National Cancer Center Japan, Tokyo, Japan

${ }^{2}$ Department of Nursing, National Cancer Center Hospital, Tokyo, Japan

${ }^{3}$ Faculty of Bioscience and Applied Chemistry, Hosei University, Koganei, Tokyo, Japan

${ }^{4}$ Department of Psychology, Faculty of Letters, Hosei University, Chiyoda-ku, Tokyo, Japan

${ }^{5}$ Division of Biostatistical Research, Center for Public Health Sciences, National Cancer Center Japan, Chuo-ku, Tokyo, Japan

${ }^{6}$ Biostatistics Division, Center for Research Administration and Support, National Cancer Center Hospital, Tokyo, Chuo-ku, Japan

${ }^{7}$ Division of Prevention, Center for Public Health Sciences, National Cancer Center Japan, Tokyo, Chuo-ku, Japan

${ }^{8}$ Cancer Solutions, Co., Ltd, Tokyo, Japan

${ }^{9}$ SUSMED, Inc, Tokyo, Japan

${ }^{10}$ Department of Breast Oncology, Aichi Cancer Center Hospital, Nagoya, Japan 
${ }^{11}$ Lifestyle Medicine, Cooperative Graduate Program, The Jikei University Graduate School of Medicine, Tokyo, Japan

Acknowledgements We are grateful to all who participated in the study interviews and shared valuable knowledge and experience regarding the planning of the survey and the preparation of the study plan. We also thank Noriko Watanabe, Noriaki Tatematsu, Hikaru Ihira, Yuri Ishino and Hiroko Noguchi for their generous support of the study.

Contributors YS, HI, EO, KT, RO, HA and YM conceived the study and drafted the original protocol. YS, HI, EO, KT, RO, HA, TS, TN and YM participated in refining the protocol. YS, KT, EO, HA, RO, TS, NS, AK, TU, HI and YM contributed to developing the survey questionnaires. AK played a major role in the statistical consideration. All authors participated in, read and approved the final manuscript.

Funding This study is supported by a National Cancer Centre Research and Development Fund (30-A-17).

Disclaimer Study enrolment and data collection are ongoing. The planned end date for this study is 31 March 2020.

Competing interests EO has received research support from Nippon Suisan Kaisha. YM has received speaker fees from Pfizer, Mochida, Eli Lilly, Morinaga Milk and NTT Data and has conducted collaborative research with Morinaga Milk. AK has received speaking fees from Chugai Pharmaceutical. All other authors declare that they have no competing interests regarding this work.

Patient consent for publication Not required.

Ethics approval The protocol was approved by the IRB of the National Cancer Center on 11 January 2019 (ID: 2018-295). In addition, many of the participating facilities required ethical approval from their local IRBs. Accordingly, the protocol was approved by the IRBs of the following facilities: Kushiro Rosai Hospital, Miyagi Cancer Centre, Nihonkai General Hospital, Saitama Medical Centre, Chiba Cancer Centre, Toranomon Hospital, Kanagawa Cancer Centre, St. Marianna University School of Medicine Hospital, Saitama Cancer Centre, Niigata Cancer Centre, Ogaki Municipal Hospital, Seirei Hamamatsu General Hospital, Aichi Cancer Centre, Mie University Hospital, Toyohashi Municipal Hospital, Ise Red Cross Hospital, Kanazawa Medical University Hospital, Yao Municipal Hospital, Osaka Breast Clinic, Tenri Hospital, Yamaguchi University Hospital, Matsuyama Red Cross Hospital, Kyushu Cancer Centre. The other facilities did not require ethical approval from their local IRBs.

Provenance and peer review Not commissioned; externally peer reviewed.

Open access This is an open access article distributed in accordance with the Creative Commons Attribution Non Commercial (CC BY-NC 4.0) license, which permits others to distribute, remix, adapt, build upon this work non-commercially, and license their derivative works on different terms, provided the original work is properly cited, appropriate credit is given, any changes made indicated, and the use is non-commercial. See: http://creativecommons.org/licenses/by-nc/4.0/.

\section{ORCID iDs}

Taichi Shimazu http://orcid.org/0000-0001-6000-9830

Yutaka Matsuoka http://orcid.org/0000-0002-8690-8129

\section{REFERENCES}

1 Hori M, Matsuda T, Shibata A, et al. Cancer incidence and incidence rates in Japan in 2009: a study of 32 population-based cancer registries for the monitoring of cancer incidence in Japan (MCIJ) project. Jpn J Clin Oncol 2015;45:884-91.

2 Denlinger CS, Sanft T, Baker KS, et al. Survivorship, version 2.2017, NCCN clinical practice guidelines in oncology. J Natl Compr Canc Netw 2017;15:1140-63.

3 Irwin ML, McTiernan A, Manson JE, et al. Physical activity and survival in postmenopausal women with breast cancer: results from the women's health Initiative. Cancer Prev Res 2011;4:522-9.

4 Tao M-H, Hainaut P, Marian C, et al. Association of prediagnostic physical activity with survival following breast cancer diagnosis: influence of TP53 mutation status. Cancer Causes Control 2013;24:2177-86.

5 Holmes MD, Chen WY, Hankinson SE, et al. Physical activity's impact on the association of fat and fiber intake with survival after breast cancer. Am J Epidemiol 2009;170:1250-6.

6 Bertram LAC, Stefanick ML, Saquib N, et al. Physical activity, additional breast cancer events, and mortality among early-stage breast cancer survivors: findings from the WHEL study. Cancer Causes Control 2011;22:427-35.

7 Beasley JM, Kwan ML, Chen WY, et al. Meeting the physical activity guidelines and survival after breast cancer: findings from the after breast cancer pooling project. Breast Cancer Res Treat 2012;131:637-43.

8 The Japan Breast Cancer Society. Relationship between lifestyle, environmental factors and prognosis of breast cancer patients CQ9. Can we recommend breast cancer patients maintain high levels of physical activity? Part of epidemiology and diagnosis. In: The Japan breast cancer Society clinical practice guidelines for breast cancer. 2018. Tokyo: Kanehara \& Co Ltd, 2018: 144-8.

9 The Japan Breast Cancer Society. Dose physical activity influence breast cancer patients' overall survival? 3rd ed. Tokyo: Kanehara \& Co., Ltd, 2015.

10 Runowicz CD, Leach CR, Henry NL, et al. American cancer Society/ American Society of clinical oncology breast cancer survivorship care guideline. CA Cancer J Clin 2016;66:43-73.

11 Caspersen CJ, Powell KE, Christenson GM. Physical activity, exercise, and physical fitness: definitions and distinctions for healthrelated research. Public Health Rep 1985;100:126-31.

12 Harrison S, Hayes SC, Newman B. Level of physical activity and characteristics associated with change following breast cancer diagnosis and treatment. Psychooncology 2009;18:387-94.

13 Tomita M, Kato T, Miyagi E, et al. Study on lifestyle and its support of gynecology cancer patients Tokyo: Foundataion for promotion of cancer research, 2017. Available: https://www.fpcr.or.jp/pdf/p13/ tomita.pdf [Accessed 6 Jun 2019].

14 Department of Food Safety,. Ministry of health, labour and welfare: The National health and nutrition survey in Japan, 2017. Available: https://www.mhlw.go.jp/stf/seisakunitsuite/bunya/kenkou_iryou/ kenkou/eiyou/h29-houkoku.html [Accessed 6 Jun 2019].

15 Hefferon $\mathrm{K}$, Murphy $\mathrm{H}$, McLeod J, et al. Understanding barriers to exercise implementation 5-year post-breast cancer diagnosis: a large-scale qualitative study. Health Educ Res 2013;28:843-56.

16 Ottenbacher AJ, Day RS, Taylor WC, et al. Exercise among breast and prostate cancer survivors - what are their barriers? J Cancer Surviv 2011;5:413-9.

17 Gielen AC ME, Gary TL, Bone LR. Using the Precede-Proceed Model To Apply Health Behavior Theories. In: Glanz KRB, Viswanath K, eds. Health promotion planning: an educational and ecological approach. 4th edn. Mountain View, CA: Mayfield Publishers, 2005.

18 Green LW, Kreuter MW. Health promotion planning; An educational and environmental approach. In: Jinba M, Iwanaga T, Matsuno T, eds. Health promotion planning; promoting activity through the Precede-Proceed model. Tokyo; Igaku Shoten. 2nd ed. California: Mayfield Publishing Company, 1991, 1997.

19 The Japan Breast Cancer Society. Accredited and related facilities, 2018. Available: http://jbcs.gr.jp/member/aboutus/shisetsu/ [Accessed 6 Feb 2019].

20 Denlinger CS, Carlson RW, Are M, et al. Survivorship: introduction and definition. clinical practice guidelines in oncology. J Nat/ Compr Canc Netw 2014;12:34-45.

21 Rock Cheryl L, Doyle C, Demark-Wahnefried W, et al. Nutrition and physical activity guidelines for cancer survivors. CA: A Cancer Journal for Clinicians 2012;62:242-74.

22 Cabinet Office, Government of Japan. Public opinion survey on cancer control, 2014. Available: https://survey.gov-online.go.jp/h26/ h26-gantaisaku/5.html [Accessed 6 Jun 2019].

23 World Health Organization. Global Physical Activity Surveillance [Available from. Available: http://www.who.int/ncds/surveillance/ steps/GPAQ/en/ [Accessed 6 Jun 2019].

24 Cleland CL, Hunter RF, Kee F, et al. Validity of the global physical activity questionnaire (GPAQ) in assessing levels and change in moderate-vigorous physical activity and sedentary behaviour. BMC Public Health 2014;14:1255.

$25 \mathrm{Chu}$ AHY, Ng SHX, Koh D, et al. Reliability and validity of the selfand Interviewer-Administered versions of the global physical activity questionnaire (GPAQ). PLoS One 2015;10:e0136944.

26 Skender S, Ose J, Chang-Claude J, et al. Accelerometry and physical activity questionnaires - a systematic review. BMC Public Health 2016:16:515

27 Murase N, Osada T, Kime R, et al. Reliability and validity of the GPAQ and IPAQ in Japanese. Japanese Journal of Physical Fitness and Sports Medicine 2004;53:899. In Japanese.

28 Inoue S, Nakata Y, Ohkawara K, et al. Development of Japanese version of the GPAQ. Japanese Journal of Physical Fitness and Sports Medicine 2016;65:155. In Japanese.

29 Brooks R. EuroQol: the current state of play. Health Policy 1996;37:53-72. 
30 Rabin R, de Charro F. EQ-5D: a measure of health status from the EuroQol group. Ann Med 2001;33:337-43.

31 Janssen MF, Pickard AS, Golicki D, et al. Measurement properties of the EQ-5D-5L compared to the EQ-5D-3L across eight patient groups: a multi-country study. Qual Life Res 2013;22:1717-27.

32 Tsuchiya A, Ikeda S, Ikegami N, et al. Estimating an EQ-5D population value set: the case of Japan. Health economics 2002;11:341-53.

33 Kessler RC, Barber C, Beck A, et al. The world Health organization health and work performance questionnaire (HPQ). J Occup Environ Med 2003;45:156-74.

34 Kessler R, Petukhova M, Mclnnes K. Japanese version of the HPQ short form (absenteeism and Presenteeism questions and scoring rules): Harvard medical school, 2007. Available: http://www.hcp.med. harvard.edu/hpq/info.php [Accessed 6 Jun 2019].

35 Kessler RC, Ames M, Hymel PA, et al. Using the world Health organization health and work performance questionnaire (HPQ) to evaluate the indirect workplace costs of illness. J Occup Environ Med 2004;46:S23-37.

36 Okuyama T, Akechi T, Kugaya A, et al. Development and validation of the cancer fatigue scale: a brief, three-dimensional, self-rating scale for assessment of fatigue in cancer patients. J Pain Symptom Manage 2000;19:5-14.

37 Vickberg SMJ. The concerns about recurrence scale (CARS): a systematic measure of women's fears about the possibility of breast cancer recurrence. Ann Behav Med 2003;25:16-24.

38 Momino K, Akechi T, Yamashita T, et al. Psychometric properties of the Japanese version of the concerns about recurrence scale (CARS-J). Jpn J Clin Oncol 2014;44:456-62.

39 Soldatos CR, Dikeos DG, Paparrigopoulos TJ. Athens insomnia scale: validation of an instrument based on ICD-10 criteria. $J$ Psychosom Res 2000;48:555-60.

40 Okajima I, Nakajima S, Kobayashi M, et al. Development and validation of the Japanese version of the Athens insomnia scale. Psychiatry Clin Neurosci 2013;67:420-5.

41 Basch E, Reeve BB, Mitchell SA, et al. Development of the National cancer Institute's patient-reported outcomes version of the common terminology criteria for adverse events (PRO-CTCAE). J Natl Cancer Inst 2014;106:dju244.

42 Dueck AC, Mendoza TR, Mitchell SA, et al. Validity and reliability of the US National cancer Institute's patient-reported outcomes version of the common terminology criteria for adverse events (PROCTCAE). JAMA Oncol 2015;1:1051-9.

43 Kawaguchi T, Azuma K, Sano M, et al. The Japanese version of the National cancer Institute's patient-reported outcomes version of the common terminology criteria for adverse events (PRO-CTCAE): psychometric validation and discordance between clinician and patient assessments of adverse events. J Patient Rep Outcomes 2017;2:2.

44 Miyaji T, lioka Y, Kuroda Y, et al. Japanese translation and linguistic validation of the US National cancer Institute's patient-reported outcomes version of the common terminology criteria for adverse events (PRO-CTCAE). J Patient Rep Outcomes 2017;1:8.

45 Zhang X, Li Y, Liu D. Effects of exercise on the quality of life in breast cancer patients: a systematic review of randomized controlled trials. Support Care Cancer 2018.

46 Oka K, Takenaka K, Miyazaki Y. Assessing the stages of change for exercise behavior among young adults : The relationship with selfreported physical activity and exercise behavior. Jpn Health Psychol 2000:17-23. In Japanese.

47 Oka K. Reliability and validity of the stages of change for exercise behavior scale among middle-aged adults. Japanese Journal of Health Promotion 2003;5:15-22. In Japanese.

48 Itakura M, Oka K, Takeda N, et al. Relationship between physical activity and social support related to exercise in adults. Walking research 2003;7:151-8. In Japanese.

49 Nakayama T. The influence of human support on exercise selfefficacy of the elderly: Focus on contents of support and subject of influence. Japanese journal of sports and health science 2013;35:99-110. In Japanese.

50 Nakayama T, Kawanishi M, Kitamura N, et al. A study on the association between approach from others and physical activity among the elderly: development and cross-validation of measurement scale. Japanese Society of Physical Education 2003;54:222. In Japanese.

51 Gjerset GM, Fossa SD, Courneya KS, et al. Interest and preferences for exercise counselling and programming among Norwegian cancer survivors. Eur J Cancer Care 2011;20:96-105.

52 Wagnild GM, Young HM. Development and psychometric evaluation of the resilience scale. J Nurs Meas 1993;1:165-78.

53 Nishi D, Uehara R, Kondo M, et al. Reliability and validity of the Japanese version of the resilience scale and its short version. BMC Res Notes 2010;3.

54 Niederdeppe J, Levy AG. Fatalistic beliefs about cancer prevention and three prevention behaviors. Cancer Epidemiology Biomarkers Prevention 2007;16:998-1003.

55 Long J. Regression models for categorical and limited dependent variables. Thousand Oaks, CA: SAGE Publications Inc, 1997. 